\title{
A STUDY OF METRIC-DEPENDENT DIMENSION FUNCTIONS $\left({ }^{1}\right)$
}

\author{
BY \\ KEIÓ NAGAMI AND J. H. ROBERTS
}

1. Introduction. This paper is a study of metric-dependent dimension functions for metric spaces. Let $X$ be a metric space with metric $\rho$. We introduced in a previous paper [15] two dimension functions $d_{1}$ and $d_{2}$ of $(X, \rho)$ which by definition appear to depend on $\rho$. We showed however, that $d_{1}(X, \rho)=\operatorname{dim} X$ (the covering dimension of $X$ ). On the other hand $d_{2}$ does depend on the particular metric $\rho$, and there exists $(X, \rho)$ with $d_{2}(X, \rho)<\operatorname{dim} X$.

Definition 1 . The empty set $\varnothing$ has $d_{2} \varnothing=-1 . d_{2}(X, \rho) \leqq n$ if $(X, \rho)$ satisfies the condition:

$\left(\mathrm{D}_{2}\right)$ For any $n+1$ pairs of closed sets $C_{1}, C_{1}^{\prime} ; \ldots ; C_{n+1}, C_{n+1}^{\prime}$ with $\rho\left(C_{i}, C_{i}^{\prime}\right)>0$, $i=1, \ldots, n+1$, there exist closed sets $B_{i}, i=1, \ldots, n+1$, such that (i) $B_{i}$ separates $C_{i}$ and $C_{i}^{\prime}$ for each $i$ and (ii) $\bigcap_{i=1}^{n+1} B_{i}=\varnothing$.

If $d_{2}(X, \rho) \leqq n$ and the statement $d_{2}(X, \rho) \leqq n-1$ is false, we set $d_{2}(X, \rho)=n$.

This definition stems from Eilenberg-Otto's characterization of dimension [3]:

A metric space $X$ has $\operatorname{dim} X \leqq n$ if and only if the following condition is satisfied:

$\left(\mathrm{D}_{2}^{\prime}\right)$ For any $n+1$ pairs of closed sets $C_{1}, C_{1}^{\prime} ; \ldots ; C_{n+1}, C_{n+1}^{\prime}$ with $C_{i} \cap C_{i}^{\prime}=\varnothing$, $i=1, \ldots, n+1$, there exist closed sets $B_{i}, i=1, \ldots, n+1$, such that (i) $B_{i}$ separates $C_{i}$ and $C_{i}^{\prime}$ for each $i$ and (ii) $\bigcap_{i=1}^{n+1} B_{i}=\varnothing$.

This characterization of (covering) dimension is still true even when $X$ is only a normal space (cf. Hemmingsen [5, Theorem 6.1] or Morita [10, Theorem 3.1]). All spaces considered in this paper are $T_{1}$. To clarify the situation of $d_{2}$ we introduce the following two apparently metric-dependent dimension functions which are similar to $d_{2}$.

Definition 2. First we set $d_{3} \varnothing=-1 . d_{3}(X, \rho) \leqq n$ if $(X, \rho)$ satisfies the condition:

$\left(\mathrm{D}_{3}\right)$ For any finite number $m$ of pairs of closed sets $C_{1}, C_{1}^{\prime} ; \ldots ; C_{m}, C_{m}^{\prime}$ with $\rho\left(C_{i}, C_{i}^{\prime}\right)>0, i=1, \ldots, m$, there exist closed sets $B_{i}, i=1, \ldots, m$, such that (i) $B_{i}$ separates $C_{i}$ and $C_{i}^{\prime}$ for each $i$ and (ii) the order of $\left\{B_{i}: i=1, \ldots, m\right\}$, ord $\left\{B_{i}\right\}$, is at most $n$.

If $d_{3}(X, \rho) \leqq n$ and the statement $d_{3}(X, \rho) \leqq n-1$ is false, then we set $d_{3}(X, \rho)=n$.

Definition 3. First we set $d_{4} \varnothing=-1 . d_{4}(X, \rho) \leqq n$ if $(X, \rho)$ satisfies the condition:

$\left(\mathrm{D}_{4}\right)$ For any countable number of pairs of closed sets $C_{1}, C_{1}^{\prime} ; C_{2}, C_{2}^{\prime} ; \ldots$ with $\rho\left(C_{i}, C_{i}^{\prime}\right)>0, i=1,2, \ldots$, there exist closed sets $B_{i}, i=1,2, \ldots$, such that (i) $B_{i}$ separates $C_{i}$ and $C_{i}^{\prime}$ for each $i$ and (ii) ord $\left\{B_{i}: i=1,2, \cdots\right\} \leqq n$.

Received by the editors January 13, 1966.

( ${ }^{1}$ This research was supported in part by the National Science Foundation Grant GP-2065. 
If $d_{4}(X, \rho) \leqq n$ and the statement $d_{4}(X, \rho) \leqq n-1$ is false, then we set $d_{4}(X, \rho)=n$.

Let $\left(D_{3}^{\prime}\right)$ (respectively $\left(D_{4}^{\prime}\right)$ ) be the condition which is obtained from $\left(D_{3}\right)$ (resp. from $\left(\mathrm{D}_{4}\right)$ ) when " $\rho\left(C_{i}, C_{i}^{\prime}\right)>0$ " is replaced by " $C_{i} \cap C_{i}^{\prime}=\varnothing$ ". It is evident that $\left(D_{4}^{\prime}\right)$ implies $\left(D_{3}^{\prime}\right)$, say $\left(D_{4}^{\prime}\right) \rightarrow\left(D_{3}^{\prime}\right)$, and $\left(D_{3}^{\prime}\right) \rightarrow\left(D_{2}^{\prime}\right)$. It is also true that $\left(D_{4}\right) \rightarrow\left(D_{3}\right)$ $\rightarrow\left(D_{2}\right)$. Morita [10] proved that $\left(D_{2}^{\prime}\right) \rightarrow\left(D_{3}^{\prime}\right) \rightarrow\left(D_{4}^{\prime}\right)$ even when $X$ is only a normal space. Then it is natural to ask whether or not $\left(D_{2}\right) \rightarrow\left(D_{3}\right) \rightarrow\left(D_{4}\right)$. The answer is no for each implication. It will be shown that $d_{4}(X, \rho)=\operatorname{dim} X$ for any $(X, \rho)$ (Theorem 2 below). Moreover we shall construct in this paper a space $(R, \rho)$ such that $d_{2}(R, \rho)=2, d_{3}(R, \rho)=3$ and $d_{4}(R, \rho)=4$. It is to be noticed that $(R, \rho)$ is topologically complete and $\rho$ is totally bounded. Our dimension functions are closely related to so-called metric dimension which is defined as follows:

Definition 4. First we set $\mu \operatorname{dim} \varnothing=-1 . \mu \operatorname{dim}(X, \rho) \leqq n$ if $(X, \rho)$ satisfies the condition:

$\left(D_{0}\right)$ There exists a sequence of open coverings $\mathscr{U}_{i}$ of $X$ such that (i) ord $\mathscr{U}_{i} \leqq n+1$ for each $i$ and (ii) mesh $\mathscr{U}_{i}\left(=\sup \left\{\rho(U): U \in \mathscr{U}_{i}\right\}\right)$ converges to zero.

If $\mu \operatorname{dim}(X, \rho) \leqq n$ and the statement $\mu \operatorname{dim}(X, \rho) \leqq n-1$ is false, then we set $\mu \operatorname{dim}(X, \rho)=n$.

Here we note that whether $\mu \operatorname{dim}(X, \rho)=\operatorname{dim} X$ or not had been a serious problem in dimension theory and that the gap between $\mu$ dim and dim played an important role when the study of dimension theory moved to general metric spaces from separable metric spaces (cf. Sitnikov [19], Nagata [17], [18], Nagami [13], Vopěnka [22], Dowker-Hurewicz [2] and Katětov [8]).

We prove that $d_{3}(X, \rho) \leqq \mu \operatorname{dim}(X, \rho)$ for any $(X, \rho)$ and that $d_{3}(X, \rho)=\mu \operatorname{dim}(X, \rho)$ when $\rho$ is totally bounded (Theorems 4 and 5 below). Thus the space $(R, \rho)$ mentioned before offers an example such that $d_{2}(R, \rho)<\mu \operatorname{dim}(R, \rho)<\operatorname{dim} R$. Sitnikov [19] was the first to construct a space $(Y, \rho)$ such that $\mu \operatorname{dim}(Y, \rho)<\operatorname{dim} Y$.

In every Cantor $n$-manifold $\left(K_{n}, \rho\right), n \geqq 3$, we shall construct subspaces $\left(X_{n}, \rho\right)$ and $\left(Y_{n}, \rho\right)$ such that

(i) $\operatorname{dim} X_{n}=\operatorname{dim} Y_{n} \geqq n-1$ and

(ii) $d_{2}\left(X_{n}, \rho\right)=\mu \operatorname{dim}\left(Y_{n}, \rho\right)=[n / 2]$.

To prove $\operatorname{dim} X_{n}$ or $\operatorname{dim} Y_{n} \geqq n-1$ we need the following theorem (Theorem 1 below) which is interesting in itself:

If $A_{i}, i=1,2, \ldots$, are disjoint closed sets of $K_{n}$ with $\operatorname{dim} A_{i} \leqq n-1$ for every $i$, then $\operatorname{dim}\left(K_{n}-\bigcup A_{i}\right) \geqq n-1$.

Sitnikov [20] proved that $\operatorname{dim}\left(K_{n}-\bigcup A_{i}\right) \geqq n-1$ if $K_{n}=I^{n}$ (n-cube) without the condition $\operatorname{dim} A_{i} \leqq n-1$ and with $A_{i} \neq I^{n}$ for $i=1,2, \ldots$ Then it is natural to ask whether our present theorem for $K_{n}$ is still true without any hypothesis on $\operatorname{dim} A_{i}$, and with $A_{i} \neq K_{n}$ for $i=1,2, \ldots$ We give a negative answer for this question. (See Figure 2.)

We give for each $n \geqq 2$ a metric space $Z_{n}$ which allows equivalent metrics $\rho_{m}$, $m=[(n+1) / 2],[(n+1) / 2]+1, \ldots, n$, such that $d_{2}\left(Z_{n}, \rho_{m}\right)=\mu \operatorname{dim}\left(Z_{n}, \rho_{m}\right)=m$. This 
space not only illustrates the dependence of $\mu$ dim and $d_{2}$ on the metric but plays a role in the construction of our final example $R$ which is mentioned above.

The final section lists four unsolved problems.

\section{Dimension of the complement of a disjoint collection of sets.}

LEMMA 1. Let $X$ be a hereditarily normal space and $Y$ a subset of $X$ with $\operatorname{dim}(X-Y)<n$. Then for any $n$ pairs of disjoint closed sets of $X, C_{1}, C_{1}^{\prime} ; \ldots ; C_{n}, C_{n}^{\prime}$, there exist closed sets of $X, B_{1}, \ldots, B_{n}$, such that $\bigcap B_{i} \subset Y$ and $B_{i}$ separates $C_{i}$ and $C_{i}^{\prime}$ for each $i$.

Proof. Let $D_{1}, D_{1}^{\prime} ; \ldots ; D_{n}, D_{n}^{\prime}$ be open sets of $X$ such that $C_{i} \subset D_{i}, C_{i}^{\prime} \subset D_{i}^{\prime}$ and $\bar{D}_{i} \cap \bar{D}_{i}^{\prime}=\varnothing$ for each $i$. By Hemmingsen [5, Theorem 6.1] or Morita [10, Theorem 3.1] there exist relatively open sets $U_{1}, \ldots, U_{n}$ of $X-Y$ such that

(i) $\bar{D}_{i}-Y \subset U_{i} \subset \bar{U}_{i}-Y \subset\left(X-\bar{D}_{i}^{\prime}\right)-Y, i=1, \ldots, n$,

(ii) $\cap\left(\bar{U}_{i}-U_{i}\right) \subset Y$.

If we set $G_{i}=C_{i} \cup U_{i}$ and $H_{i}=C_{i}^{\prime} \cup\left((X-Y)-\bar{U}_{i}\right)$, then $\bar{G}_{i} \cap H_{i}=G_{i} \cap \bar{H}_{i}=\varnothing$. By the hereditary normality of $X$ there exists an open set $V_{i}$ of $X$ such that $G_{i} \subset V_{i} \subset \bar{V}_{i} \subset X-H_{i}$. Set $B_{i}=\bar{V}_{i}-V_{i}$. Then $B_{i}, i=1, \ldots, n$, satisfy the required condition.

LEMMA 2. Let $X$ be a compact Hausdorff space and let $H$ and $K$ be disjoint closed sets of $X$ such that no connected set meets both $H$ and $K$. Then there exist disjoint open sets $H_{1}$ and $K_{1}$ such that $H \subset H_{1}, K \subset K_{1}$ and $H_{1} \cup K_{1}=X$.

This can be proved by a method analogous to the one in Moore [9, Theorem 44, p. 15] with the consideration of Hocking-Young [6, Theorem 2-9, p. 44].

LEMMA 3. A connected compact Hausdorff space cannot be decomposed into a countably infinite or finite (but more than one) union of disjoint closed subsets.

This can be proved by a method analogous to the one in Moore [9, Theorem 56, p. 23] with the aid of Lemma 2.

Definition 5. Let $X$ be a normal space. A system of pairs $C_{1}, C_{1}^{\prime} ; \ldots ; C_{n}, C_{n}^{\prime}$ is called a defining system of $X$ if (i) $C_{i}$ and $C_{i}^{\prime}$ are disjoint closed sets of $X$ for each $i$ and (ii) for arbitrary closed sets $B_{i}, i=1, \ldots, n$, separating $C_{i}$ and $C_{i}^{\prime}$ we have $\bigcap B_{i} \neq \varnothing$.

LEMMA 4. Let $X$ be a compact Hausdorff space, $F$ a closed set of $X$ and $f$ a mapping (continuous transformation) of $F$ into the $(n-1)$-sphere $S^{n-1}$. Consider $S^{n-1}$ as the surface of the $n$-cube $I^{n}=\left\{\left(x_{1}, \ldots, x_{n}\right):-1 \leqq x_{i} \leqq 1\right\}$. Let $C_{1}, C_{1}^{\prime} ; \ldots ; C_{n}, C_{n}^{\prime}$ be $n$ pairs of opposite faces of $I^{n}$ defined by:

$$
C_{i}=\left\{\left(x_{1}, \ldots, x_{n}\right): x_{i}=-1\right\}, \quad C_{i}^{\prime}=\left\{\left(x_{1}, \ldots, x_{n}\right): x_{i}=1\right\},
$$

$i=1, \ldots, n$. If the system $f^{-1}\left(C_{1}\right), f^{-1}\left(C_{1}^{\prime}\right) ; \ldots ; f^{-1}\left(C_{n}\right), f^{-1}\left(C_{n}^{\prime}\right)$ is not defining, then $f$ has an extension $f^{*}: X \rightarrow S^{n-1}$. 
Proof. Let $B_{1}, \ldots, B_{n}$ be closed sets of $X$ such that $B_{i}$ separates $f^{-1}\left(C_{i}\right)$ and $f^{-1}\left(C_{i}^{\prime}\right)$ for every $i$ and such that $\bigcap B_{i}=\varnothing$. By Morita [10, Lemma 1.2] we can assume that every $B_{i}$ is a $G_{\delta}$. Let $f(x)=\left(f_{1}(x), \ldots, f_{n}(x)\right)$, where each $f_{i}$ is a mapping into $[-1,1]$. Let $g_{i}: X \rightarrow[-1,1]$ be an extension of $f_{i} \mid f^{-1}\left(C_{i}\right) \cup f^{-1}\left(C_{i}^{\prime}\right)$ such that $g_{i}(x)=0$ if and only if $x \in B_{i}$ and such that $\left|g_{i}(x)\right|=1$ if and only if $x \in f^{-1}\left(C_{i}\right)$ $\cup f^{-1}\left(C_{i}^{\prime}\right)$. Let $g(x)=\left(g_{1}(x), \ldots, g_{n}(x)\right)$. Then $g$ is a mapping of $X$ into $I^{n}$ and $g(F) \subset S^{n-1}$. If $x \in F$, then $f(x)$ and $g(x)$ cannot be a pair of opposite points on $S^{n-1}$. Hence $f$ is homotopic to $g \mid F$. Let $p$ be the original point $(0, \ldots, 0)$ of $I^{n}$. Then $p \notin g(X)$. Let $r: I^{n}-p \rightarrow S^{n-1}$ be a retraction. Then $r g$ maps $X$ into $S^{n-1}$. By the same argument as in Hurewicz-Wallman [7, Chapter VI], $f$ has an extension $f^{*}$ over $X$ whose values are still in $S^{n-1}$.

THEOREM 1. Let $X$ be a compact hereditarily normal space with $\operatorname{dim} X=n, n \geqq 1$, and $A_{1}, A_{2}, \cdots$ be a sequence of disjoint closed sets of $X$ such that $\operatorname{dim} A_{1} \leqq n-1$ for each $i$. Then

$$
\operatorname{dim}\left(X-\cup A_{i}\right) \geqq n-1 .
$$

Proof. First step. Since $\operatorname{dim} X=n$ there exist by Morita [10, Theorem 5.1] a closed set $F$ of $X$, a mapping $f$ of $F$ into $S^{n-1}$ and a closed set $Y \supset F$ such that (i) $f$ cannot be extended over $Y$ and (ii) if $Z$ is any proper closed subset of $Y$ with $F \subset Z$, then $f$ is extendable over $Z$. We may even assume that $Y$ is actually a Cantor $n$-manifold (cf. Hurewicz-Wallman [7, pp. 99-100]). Since $\operatorname{dim}\left(Y-\cup A_{i}\right) \geqq n-1$ implies $\operatorname{dim}\left(X-\cup A_{i}\right) \geqq n-1$, we assume hereafter $X$ is $Y$ itself with the above minimal property.

Second step. Since $\operatorname{dim} A_{1} \leqq n-1$, there exists a mapping $f^{\prime}: F \cup A_{1} \rightarrow S^{n-1}$ with $f^{\prime} \mid F=f$. Since $S^{n-1}$ is a neighborhood extensor for normal spaces by Hanner [4, Theorem 13.2], there exist an open set $U_{1}$ with $U_{1} \supset F \cup A_{1}$ and a mapping $f_{1}: \bar{U}_{1} \rightarrow S^{n-1}$ with $f_{1} \mid F \cup A_{1}=f^{\prime}$. Continuing such procedure, we have a sequence of open sets $U_{1}, U_{2}, \cdots$ and a sequence of mappings $f_{i}: \bar{U}_{i} \rightarrow S^{n-1}$ such that (i) $F \cup\left(\bigcup_{i \leqq i} A_{i}\right) \subset U_{i}$ and $\bar{U}_{i} \subset U_{i+1}$, for every $i$ and (ii) $f_{i}$ is an extension of $f_{i-1}$ for every $i$, where $f_{0}=f$.

Define $g: \cup U_{i} \rightarrow S^{n-1}$ in such a way that $g \mid \bar{U}_{i}=f_{i}$ for each $i$. Then $g$ is an extension of $f$ over $\bigcup U_{i}$. Let $\varphi: X \rightarrow[0,1]$ be a mapping such that

(i) $\varphi(x)=0$ if and only if $x \notin \cup U_{i}$,

(ii) $\varphi(x)=1$ if $x \in F$,

(iii) $\bar{U}_{i} \subset\left\{x: \varphi(x)>2^{-i}\right\} \subset U_{i+1}$ for every $i$.

Consider $S^{n-1}$ as the surface of the solid $n$-ball $I^{n}$ of radius 1 whose center is the origin $p$. We define $h: X \rightarrow I^{n}$ as follows:

(i) $h(x)=p$ if $x \notin \bigcup U_{i}$,

(ii) $h(x)=\varphi(x) g(x)$ if $x \in \bigcup U_{i}$, where $g(x)$ is considered as a vector from $p$ to $g(x)$.

Then $h$ is continuous and $h \mid F=f$. Moreover $h^{-1}(p) \cap\left(\bigcup A_{i}\right)=\varnothing$, which will be a meaningful fact later. 
Third step. Here we reconsider that $I^{n}$ is the $n$-cube expressed as

$$
\left\{\left(x_{1}, \ldots, x_{n}\right):-1 \leqq x_{i} \leqq 1, i=1, \ldots, n\right\}
$$

whose surface is $S^{n-1}$ and whose origin $(0, \ldots, 0)$ is $p$. Consider the solid pyramid $P$ in $I^{n}$ whose base is $B=\left\{\left(x_{1}, \ldots, x_{n}\right): x_{n}=-1\right\}$ and whose apex is $p$. The $n-1$ pairs of opposite sides of $P$ may be denoted by $\left(S_{i}, T_{i}\right), i=1, \ldots, n-1$, where $S_{i}$ is spanned by

$$
S_{i}^{\prime}=\left\{\left(x_{1}, \ldots, x_{n}\right): x_{i}=x_{n}=-1\right\}
$$

and $p$, and $T_{i}$ is spanned by

$$
T_{i}^{\prime}=\left\{\left(x_{1}, \ldots, x_{n}\right): x_{i}=1, x_{n}=-1\right\}
$$

and $p$. Then

$$
C_{i}=h^{-1}\left(S_{i}\right)-h^{-1}(p), \quad C_{i}^{\prime}=h^{-1}\left(T_{i}\right)-h^{-1}(p), \quad i=1, \ldots, n-1,
$$

are $n-1$ pairs of disjoint closed sets of $X^{\prime}=X-h^{-1}(p)$. Figure 1 will help us to treat the situation.

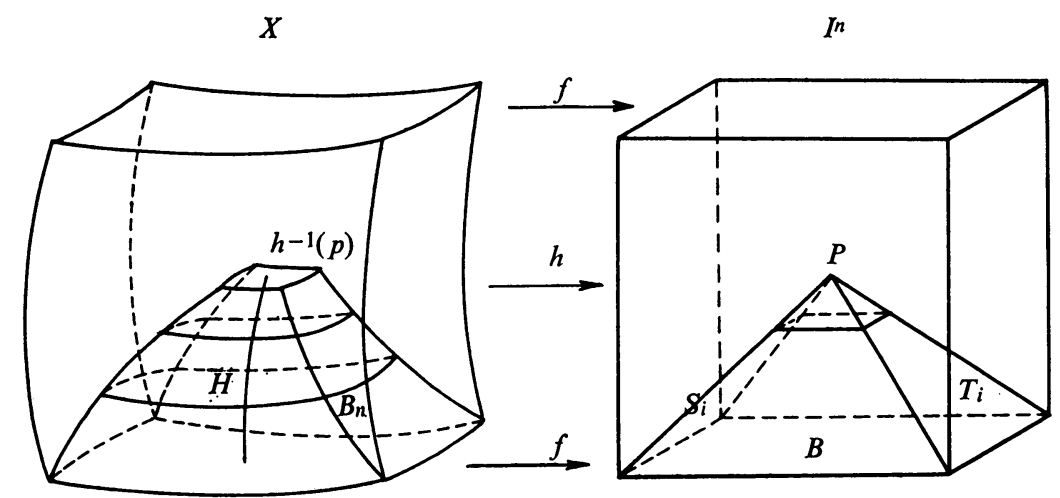

FIGURE 1

Fourth step. Assume that $\operatorname{dim}\left(X-\cup A_{i}\right)<n-1$. Then

$$
\operatorname{dim}\left(X-\left(\left(\cup A_{i}\right) \cup h^{-1}(p)\right)\right)<n-1,
$$

since $h^{-1}(p)$ is a $G_{\delta}$. By Lemma 1 there exist closed sets $B_{i}$ of $X-h^{-1}(p), i=1, \ldots$, $n-1$, such that (i) $B_{i}$ separates $C_{i}$ and $C_{i}^{\prime}$ for each $i$ and (ii)

$$
\left(\bigcap_{i=1}^{n-1} B_{i}\right) \cap\left(X-\bigcup A_{i}\right)=\varnothing \text {. }
$$

The latter condition implies $H=\bigcap_{i=1}^{n-1} B_{i} \subset \bigcup A_{i}$. Let us consider the compact set $H \cup h^{-1}(p)$ and the two disjoint subsets $H \cap h^{-1}(B)$ and $h^{-1}(p)$. Suppose that $H \cap h^{-1}(B) \neq \varnothing$ and there exists a connected closed set $K \subset H \cup h^{-1}(p)$ such that 
$K \cap H \cap h^{-1}(B) \neq \varnothing$ and $h^{-1}(p) \cap K \neq \varnothing$. Then for some $i, K \cap A_{i} \neq \varnothing$. Since $K \subset h^{-1}(p) \cup A_{1} \cup A_{2} \cup \cdots$, we have a contradiction by Lemma 3.

Fifth step. By Lemma 2 we can now conclude that there exist disjoint compact sets $H_{1}$ and $H_{2}$ such that (i) $H_{1} \cup H_{2}=h^{-1}(B) \cup H \cup h^{-1}(p)$, (ii) $h^{-1}(p) \subset H_{1}$ and (iii) $h^{-1}(B) \subset H_{2}$, whether $H \cap h^{-1}(B)=\varnothing$ or not. Hence there exists a closed set $B_{n}$ of $X$ separating $h^{-1}(p)$ and $h^{-1}(B)$ without touching $H$. Let $c$ be a number with $0<c<1, Q_{c}$ the intersection of $P$ and the hyperplane $\left\{\left(x_{1}, \ldots, x_{n}\right): x_{n}=-c\right\}, P_{c}$ the intersection of $P$ and $\left\{\left(x_{1}, \ldots, x_{n}\right): x_{n} \leqq-c\right\}$ and $R_{c}$ the surface of $P_{c}$. Then there exists a number $b$ with $0<b<1$ such that

$$
h^{-1}\left(\overline{P-P_{b}}\right) \cap B_{n}=\varnothing .
$$

If we confine our attention to the set $h^{-1}\left(P_{b}\right)$, there are closed sets

$$
B_{1} \cap h^{-1}\left(P_{b}\right), \ldots, B_{n} \cap h^{-1}\left(P_{b}\right)
$$

which separate pairs

$$
\begin{gathered}
h^{-1}\left(S_{1} \cap P_{b}\right), h^{-1}\left(T_{1} \cap P_{b}\right) ; \ldots ; h^{-1}\left(S_{n-1} \cap P_{b}\right), h^{-1}\left(T_{n-1} \cap P_{b}\right) ; \\
h^{-1}(B), h^{-1}\left(Q_{b}\right),
\end{gathered}
$$

respectively. Denote this system of pairs by $\alpha$. Since

$$
\bigcap_{i=1}^{n}\left(B_{i} \cap h^{-1}\left(P_{b}\right)\right) \subset \bigcap_{i=1}^{n} B_{i}=\varnothing,
$$

$\alpha$ is not defining. Then by Lemma 4 there exists a mapping $k_{1}: h^{-1}\left(P_{b}\right) \rightarrow R_{b}$ such that $k_{1}\left|h^{-1}\left(R_{b}\right)=h\right| h^{-1}\left(R_{b}\right)$. Let $k: X \rightarrow I^{n}$ be a mapping such that

(i) $k\left|X-h^{-1}\left(P_{b}\right)=h\right| X-h^{-1}\left(P_{b}\right)$,

(ii) $k \mid h^{-1}\left(P_{b}\right)=k_{1}$.

Let $s$ be an inner point of $P_{b}$ and $r$ a retraction of $I^{n}-\{s\}$ onto $S^{n-1}$. Then $r k: X \rightarrow S^{n-1}$ is an extension of $f$, a contradiction. Thus we have $\operatorname{dim}\left(X-\cup A_{i}\right)$ $\geqq n-1$ and the proof is completed.

Corollary 1 (Sitnikov [20]). Let $n \geqq 1$. Let $A_{i}, i=1,2, \ldots$, be a disjoint sequence of closed sets of $I^{n}$ at least two of which are not empty. Then

$$
\operatorname{dim}\left(I^{n}-\bigcup A_{i}\right) \geqq n-1 .
$$

Proof. Let $S^{n-1}$ be the surface of $I^{n}=\left\{\left(x_{1}, \ldots, x_{n}\right):-1 \leqq x_{i} \leqq 1, i=1, \ldots, n\right\}$. Let $f: S^{n-1} \rightarrow S^{n-1}$ be the identity mapping. Since it is impossible that $I^{n}-S^{n-1}$ is contained in one $A_{i}$, we have one of the following two cases:

(i) There exists $i$ such that $\left(I^{n}-S^{n-1}\right)-A_{i} \neq \varnothing$ and $A_{j} \subset S^{n-1}$ for any $j \neq i$.

(ii) There exist $i$ and $j$ with $i \neq j$ such that

$$
\left(I^{n}-S^{n-1}\right) \cap A_{i} \neq \varnothing \text { and }\left(I^{n}-S^{n-1}\right) \cap A_{j} \neq \varnothing .
$$


The first case yields $\operatorname{dim}\left(I^{n}-\bigcup A_{i}\right)=n$. If the second case happens, then there exists a number $\varepsilon$ with $0<\varepsilon<1$ such that

$$
I_{\varepsilon}^{n}=\left\{\left(x_{1}, \ldots, x_{n}\right):\left|x_{i}\right| \leqq \varepsilon, i=1, \ldots, n\right\}
$$

meets $A_{i}$ and $A_{j}$. Then by Lemma 3 there exists a point $q$ in $I_{\varepsilon}^{n}-\bigcup A_{i}$. Then we can apply the same argument on $f$ and $q$ as in the proof of Theorem 1 and we get $\operatorname{dim}\left(I^{n}-\bigcup A_{i}\right) \geqq n-1$.

COROLlaRY 2. Let $X$ be a connected metric space such that every point has a neighborhood homeomorphic to $I^{n}, n \geqq 1$. Let $A_{i}, i=1,2, \ldots$, be a disjoint sequence of closed sets of $X$ at least two of which are not empty. Then

$$
\operatorname{dim}\left(X-\bigcup A_{i}\right) \geqq n-1 \text {. }
$$

Proof. Consider a closed covering $\left\{F_{\alpha}\right\}$ of $X$ such that each $F_{\alpha}$ is homeomorphic to $I^{n}$. If each $F_{\alpha}$ is contained in some $A_{\mathfrak{i}}$, then each $A_{\mathfrak{i}}$ has to be open, which contradicts the fact that $X$ is connected. Hence (i) there exists $F_{\alpha}$ which meets at least two of the $A_{i}$ 's, (ii) there exists $F_{\beta}$ such that $F_{\beta} \cap A_{i} \neq \varnothing, F_{\beta}-A_{i} \neq \varnothing$ and $F_{\beta} \cap A_{j}=\varnothing$ for $j \neq i$, or (iii) there exists $F_{y}$ such that $F_{y} \cap A_{i}=\varnothing$ for every $i$. The first case yields $\operatorname{dim}\left(X-\bigcup A_{i}\right) \geqq \operatorname{dim}\left(F_{\alpha}-\bigcup A_{i}\right) \geqq n-1$. The second case yields $\operatorname{dim}\left(X-\cup A_{i}\right) \geqq \operatorname{dim}\left(F_{\beta}-A_{i}\right) \geqq n-1$. The third case yields $\operatorname{dim}\left(X-\cup A_{i}\right)$ $\geqq \operatorname{dim} F_{y} \geqq n-1$.

Figure 2 gives a Cantor 2-manifold $X$ such that a proposition for $X$ analogous to Corollary 1 fails. In fact $\operatorname{dim} X=2$, yet $\operatorname{dim}\left(X-\bigcup A_{i j}\right)=0$ since $X-\bigcup A_{i j}$ is a subset of the Cantor discontinuum.

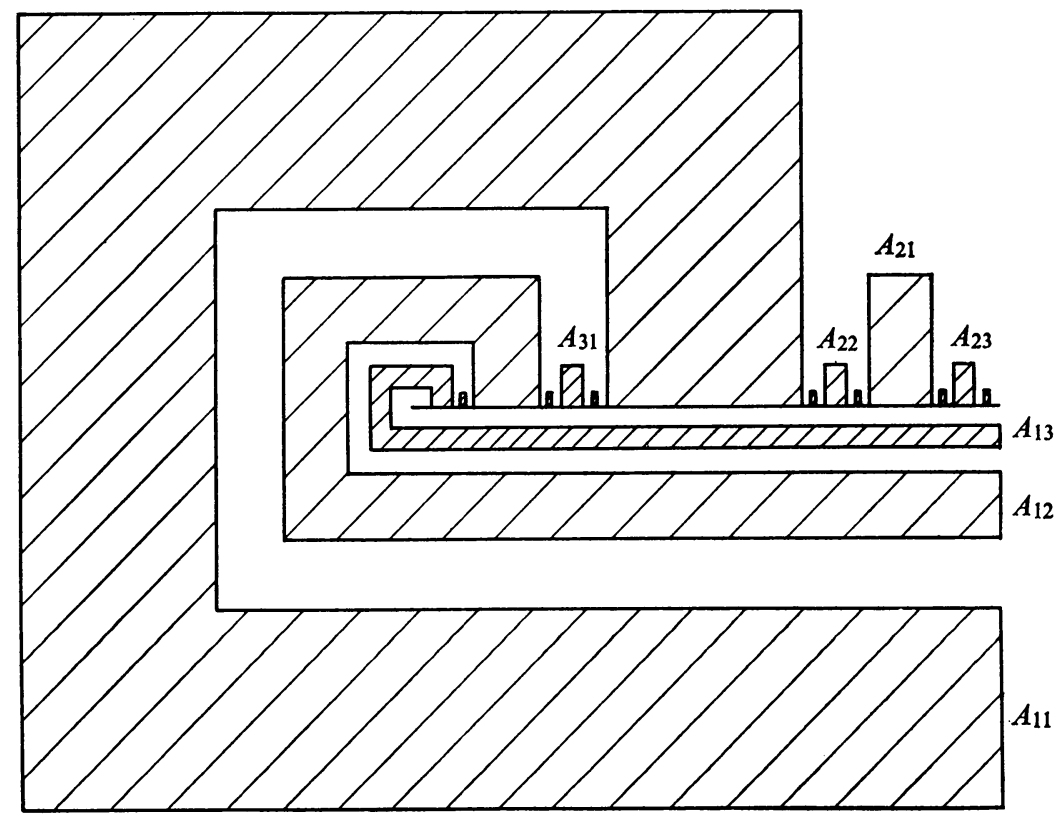

FIGURE 2 


\section{Relations among the various functions.}

Lemma 5 (MORITA [10, TheOREM 3.4]). Let $X$ be a normal space with $\operatorname{dim} X \leqq n$. Then $X$ satisfies the condition $\left(\mathrm{D}_{4}^{\prime}\right)$.

LEMMA 6. If a metric space $X$ has a o-locally finite open base $\mathscr{U}$ such that ord $\{\bar{U}-U: U \in \mathscr{U}\} \leqq n$, then $\operatorname{dim} X \leqq n$. ( $\mathscr{U}$ is called $\sigma$-locally finite if $\mathscr{U}$ can be decomposed into a countable number of locally finite subcollections.)

This is a slight modification of Morita [12, Theorem 8.7].

Theorem 2. $\operatorname{dim} X=d_{4}(X, \rho)$ for any $(X, \rho)$.

Proof. By Lemma $5 \operatorname{dim} X \geqq d_{4}(X, \rho)$. To prove $\operatorname{dim} X \leqq d_{4}(X, \rho)$ assume $d_{4}(X, \rho) \leqq n$. Let us show the existence of such $\mathscr{U}$ as in Lemma 6. By Stone [21] there exists an open base $\mathscr{V}=\bigcup_{i=1}^{\infty} \mathscr{V}_{i}$ of $X, \mathscr{V}_{i}=\left\{V_{\alpha}: \alpha \in \Lambda_{i}\right\}, i=1,2, \ldots$, such that each $\mathscr{V}_{i}$ is discrete. $\mathscr{V}_{i}$ is called discrete if $\bar{V}_{i}=\left\{\overline{\mathscr{V}_{\alpha}}: \alpha \in \Lambda_{i}\right\}$ is a locally finite disjoint collection. Set $V_{i}=\bigcup\left\{V_{\alpha}: \alpha \in \Lambda_{i}\right\}, i=1,2, \ldots$, and

$$
F_{i j}=\left\{x: \rho\left(x, X-V_{i}\right) \geqq 1 / j\right\}, \quad j=1,2, \ldots
$$

Then by $d_{4}(X, \rho) \leqq n$ there exist open sets $U_{i j}, i, j=1,2, \ldots$, such that

(i) $V_{i} \supset \bar{U}_{i j} \supset U_{i j} \supset F_{i j}$ for each $i$ and $j$ and

(ii) ord $\left\{\bar{U}_{i j}-U_{i j}: i, j=1,2, \cdots\right\} \leqq n$.

Set

$$
\mathscr{U}_{i j}=\left\{V_{\alpha} \cap U_{i j}: \alpha \in \Lambda_{i}\right\}
$$

Then $\mathscr{U}_{i j}$ is discrete and hence locally finite. $\mathscr{U}=\bigcup_{i, j=1}^{\infty} \mathscr{U}_{i j}$ is an open base for $X$ such that ord $\{\bar{U}-U: U \in \mathscr{U}\} \leqq n$. Hence by Lemma 6 we have $\operatorname{dim} X \leqq n$ and the theorem is proved.

LEMMA 7. If $\left\{F_{\alpha}\right\}$ is a locally finite closed collection of a paracompact Hausdorff space, then there exists an open collection $\left\{G_{\alpha}\right\}$ such that (i) $G_{\alpha} \supset F_{\alpha}$ for each $\alpha$ and (ii) ord $\left\{F_{\alpha}\right\}=$ ord $\left\{G_{\alpha}\right\}$.

This can easily be seen with the aid of Morita [11, Theorem 1.3].

THeOREM 3 (KATĚTov [8]). $\operatorname{dim} X \leqq 2 \mu \operatorname{dim}(X, \rho)$ for any $(X, \rho)$.

Proof. Suppose $\mu \operatorname{dim}(X, \rho) \leqq n$. Let $\mathscr{U}_{i}=\left\{U_{\alpha}: \alpha \in \Lambda_{i}\right\}, i=1,2, \ldots$, be a sequence of open coverings of $X$ such that

(i) mesh $\mathscr{U}_{i}<2^{-i}$ for each $i$ and

(ii) ord $\mathscr{U}_{i} \leqq n+1$ for each $i$.

By an easy observation we can assume that each $\mathscr{U}_{i}$ is locally finite. Let $\mathscr{G}=\left\{G_{1}, \ldots, G_{m}\right\}$ be an arbitrary finite open covering of $X$. Set

$$
D_{i}=\bigcup\left\{\left\{x: \rho\left(x, X-G_{j}\right)>2^{-i+1}\right\}: j=1, \ldots, m\right\}, \quad i=1,2, \ldots
$$

Then (i) $D_{1} \subset \bar{D}_{1} \subset D_{2} \subset \bar{D}_{2} \subset D_{3} \cdots$, (ii) each $D_{i}$ is open and (iii) $\bigcup_{i=1}^{\infty} D_{i}=X$. Let 
$\mathscr{F}_{i}=\left\{F_{\alpha}: \alpha \in \Lambda_{i}\right\}$ be a closed covering of $X$ such that $F_{\alpha} \subset U_{\alpha}$ for each $\alpha \in \Lambda_{i}$. Set

$$
\begin{aligned}
& \Lambda_{i}^{\prime}=\left\{\alpha: F_{\alpha} \cap \bar{D}_{i}=\varnothing\right\}, \\
& W_{i}=X-\bigcup\left\{F_{\alpha}: \alpha \in \Lambda_{i}^{\prime}\right\} .
\end{aligned}
$$

Then $W_{i}$ is an open set with $\bar{D}_{i} \subset W_{i} \subset \bar{W}_{i} \subset D_{i+1}$ for every $i$. Since every point of $\bar{W}_{i}-W_{i}$ is contained in some $F_{\alpha} \in \mathscr{F}_{i}$ with $\alpha \in \Lambda_{i}^{\prime}$, ord $\left\{F_{\alpha} \cap\left(\bar{W}_{i}-W_{i}\right): \alpha \notin \Lambda_{i}^{\prime}\right\} \leqq n$. (This type of argument comes from Morita [10, Theorem 3.3].) Since $\left\{F_{\alpha}: \alpha \in \Lambda_{i}-\Lambda_{i}^{\prime}\right\}$ covers $\bar{W}_{i}-W_{i}$, there exists by Lemma 7 an open collection $\mathscr{V}_{i}=\left\{V_{\alpha}: \alpha \in \Lambda_{i}-\Lambda_{i}^{\prime}\right\}$ of $X$ such that

(i) $F_{\alpha} \cap\left(\bar{W}_{i}-W_{i}\right) \subset V_{\alpha} \subset\left(D_{i+1}-\bar{D}_{i}\right) \cap U_{\alpha}$ for each $\alpha \in \Lambda_{i}-\Lambda_{i}^{\prime}$ and

(ii) ord $\left\{V_{\alpha}: \alpha \in \Lambda_{i}-\Lambda_{i}^{\prime}\right\} \leqq n$.

Then $\mathscr{V}_{i}$ refines $\mathscr{G}$. Moreover $V \in \mathscr{V}_{i}, V^{\prime} \in \mathscr{V}_{i}, i \neq j$, imply $V \cap V^{\prime}=\varnothing$. We set

$$
\mathscr{H}_{i}=\left\{U_{\alpha} \cap\left(W_{i}-\bar{W}_{i-1}\right): \alpha \in \Lambda_{i}\right\} \text {, where } W_{-1}=\varnothing .
$$

Then $H \in \mathscr{H}_{i}, H^{\prime} \in \mathscr{H}_{j}, i \neq j$, imply $H \cap H^{\prime}=\varnothing$. If we set

$$
\mathscr{H}=\left(\bigcup_{i=1}^{\infty} \mathscr{H}_{i}\right) \cup\left(\bigcup_{i=1}^{\infty} \mathscr{V}_{i}\right),
$$

then it is easy to see that $\mathscr{H}$ is an open covering of $X$ such that (i) $\mathscr{H}$ refines $\mathscr{G}$ and (ii) ord $\mathscr{H} \leqq 2 n+1$. Thus we have $\operatorname{dim} X \leqq 2 n$ and the theorem is proved.

THEOREM 4. $d_{3}(X, \rho) \leqq \mu \operatorname{dim}(X, \rho)$ for any $(X, \rho)$.

Proof. Suppose $\mu \operatorname{dim}(X, \rho) \leqq n$. Let $C_{1}, C_{1}^{\prime} ; \cdots ; C_{m}, C_{m}^{\prime}$ be a finite number of pairs of closed sets of $X$ such that there exists a positive number $\varepsilon$ such that $\rho\left(C_{i}, C_{i}^{\prime}\right)>\varepsilon$ for each $i$. By $\mu \operatorname{dim}(X, \rho) \leqq n$ there exists a locally finite open covering $\mathscr{U}=\left\{U_{\alpha}: \alpha \in \Lambda\right\}$ of $X$ such that (i) ord $\mathscr{U} \leqq n+1$ and (ii) mesh $\mathscr{U}<\varepsilon / 2$. Let $\mathscr{F}=\left\{F_{\alpha}: \alpha \in \Lambda\right\}$ be a locally finite closed covering of $X$ such that $F_{\alpha} \subset U_{\alpha}$ for each $\alpha \in \Lambda$. Set

$$
\begin{aligned}
& \Lambda^{\prime}=\left\{\alpha: F_{\alpha} \cap C_{1}=\varnothing\right\} \\
& W=X-\bigcup\left\{F_{\alpha}: \alpha \in \Lambda^{\prime}\right\} \\
& B_{1}=\bar{W}-W .
\end{aligned}
$$

Then $B_{1}$ separates $C_{1}$ and $C_{1}^{\prime}$ and

$$
\mathscr{U}_{1}=\left\{U_{\alpha}: \alpha \in \Lambda-\Lambda^{\prime}\right\} \cup\left\{U_{\alpha}-B_{1}: \alpha \in \Lambda^{\prime}\right\}
$$

is an open covering of $X$ such that

(i) $\mathscr{U}_{1}$ refines $\mathscr{U}$,

(ii) $\mathscr{U}\left|X-B_{1}=\mathscr{U}_{1}\right| X-B_{1}$,

(iii) ord $\left(x, \mathscr{U}_{1}\right) \leqq$ ord $(x, \mathscr{U})-1$ for each $x \in B_{1}$, where ord $\left(x, \mathscr{U}_{1}\right)$ is the order of $\mathscr{U}_{1}$ at $x$. 
This can be verified by the same argument as in the proof of the previous theorem. Continuing this procedure, we get closed sets $B_{i}, i=2, \ldots, m$, separating $C_{i}$ and $C_{i}^{\prime}$ respectively and open coverings $\mathscr{U}_{2}, \ldots, \mathscr{U}_{m}$ such that

(i) $\mathscr{U}_{i+1}$ refines $\mathscr{U}_{i}$ for $i=1, \ldots, m-1$,

(ii) $\mathscr{U}_{i}\left|X-B^{i}=\mathscr{U}_{i-1}\right| X-B_{i}$ for each $i$,

(iii) ord $\left(x, \mathscr{U}_{i}\right) \leqq$ ord $\left(x, \mathscr{U}_{i-1}\right)-1$ for each $x \in B_{i}, i=2, \ldots, m$.

If $x \in B_{i_{1}} \cap \cdots \cap B_{i_{n+1}}, i_{1}<i_{2}<\cdots<i_{n+1}$, then

$$
\begin{aligned}
1 & \leqq \operatorname{ord}\left(x, \mathscr{U}_{i_{n}+1}\right) \leqq \operatorname{ord}\left(x, \mathscr{U}_{i_{n+1}-1}\right)-1 \\
& \leqq \operatorname{ord}\left(x, \mathscr{U}_{i_{n}}\right)-1 \leqq \operatorname{ord}\left(x, \mathscr{U}_{i_{n}-1}\right)-2 \\
& \leqq \cdots \leqq \operatorname{ord}\left(x, \mathscr{U}_{i_{1}}\right)-n \leqq \operatorname{ord}(x, \mathscr{U})-(n+1)
\end{aligned}
$$

Thus we have ord $(x, \mathscr{U}) \geqq n+2$, a contradiction. We have therefore

$$
\text { ord }\left\{B_{i}: i=1, \ldots, m\right\} \leqq n,
$$

and the theorem is proved.

TheOREM 5. $d_{3}(X, \rho)=\mu \operatorname{dim}(X, \rho)$ for a totally bounded metric space $(X, \rho)$.

Proof. Suppose that $d_{3}(X, \rho) \leqq n$. For an arbitrary positive number $\varepsilon$ there exists a finite set of points $x_{1}, \ldots, x_{m}$ such that

$$
\left\{U_{i}=S_{\varepsilon}\left(x_{i}\right): i=1, \ldots, m\right\}
$$

covers $X$. Set

$$
V_{i}=S_{2 \varepsilon}\left(x_{i}\right), \quad i=1, \ldots, m .
$$

Then there exist open sets $W_{1}, \ldots, W_{m}$ such that

(i) $\bar{U}_{i} \subset W_{i} \subset \bar{W}_{i} \subset V_{i}$ for each $i$,

(ii) $B_{i}=\bar{W}_{i}-W_{i}$ separates $X-V_{i}$ and $\bar{U}_{i}$ for each $i$,

(iii) ord $\left\{B_{i}: i=1, \ldots, m\right\} \leqq n$.

By Lemma 7 there exist open sets $G_{1}, \ldots, G_{m}$ of $X$ such that (i) $B_{i} \subset G_{i} \subset V_{i}$ for each $i$ and (ii) ord $\left\{G_{i}\right\} \leqq n$. Set

$$
\begin{aligned}
& \mathscr{W}_{i}=\left\{W_{i 1}=W_{i}, W_{i 2}=X-\bar{W}_{i}\right\}, \\
& \mathscr{W}=\bigwedge_{i=1}^{m} \mathscr{W}_{i}=\left\{W_{1 i_{1}} \cap \cdots \cap W_{m i_{m}}: i_{1}, \ldots, i_{m}=1,2\right\} .
\end{aligned}
$$

Since

$$
\bigcap_{i=1}^{m} W_{12}=\bigcap_{i=1}^{m}\left(X-\bar{W}_{i}\right)=X-\bigcup_{i=1}^{m} \bar{W}_{i}=X-X=\varnothing,
$$

$\mathscr{W}$ refines $\left\{W_{1}, \ldots, W_{m}\right\}$ and hence $\mathscr{W}$ refines $\left\{V_{1}, \ldots, V_{m}\right\}$. Moreover

$$
\bigcup\{W: W \in \mathscr{W}\}=X-\bigcup_{i=1}^{m} B_{i}
$$

and ord $\mathscr{W} \leqq 1$. If we set

$$
\mathscr{G}=\mathscr{W} \cup\left\{G_{1}, \ldots, G_{m}\right\}
$$


then $\mathscr{G}$ is an open covering of $X$ such that (i) mesh $\mathscr{G} \leqq 4 \varepsilon$ and (ii) ord $\mathscr{G} \leqq n+1$. Thus we have $\mu \operatorname{dim}(X, \rho) \leqq n$. If we combine $d_{3}(X, \rho) \geqq \mu \operatorname{dim}(X, \rho)$ just proved with Theorem 4, we get $d_{3}(X, \rho)=\mu \operatorname{dim}(X, \rho)$ and the theorem is proved.

Since $d_{2}(X, \rho) \leqq d_{3}(X, \rho) \leqq d_{4}(X, \rho)$ are trivially true, we have now $d_{2} \leqq d_{3}$ $\leqq \mu \operatorname{dim} \leqq d_{4}=\operatorname{dim} \leqq 2 \mu \operatorname{dim}$, symbolically.

REMARK 1. When $(X, \rho)$ is locally compact, all of these dimension functions coincide with each other, of course. On the other hand there exists a space $(X, \rho)$ which is not locally compact at any point yet $d_{2}(X, \rho)=d_{3}(X, \rho)=\mu \operatorname{dim}(X, \rho)$ $=\operatorname{dim} X$. Let us consider $I^{3}=\left\{\left(x_{1}, x_{2}, x_{3}\right): 0 \leqq x_{i} \leqq 1, i=1,2,3\right\}$. Let $\rho$ be a metric of $I^{3}$. Set $B=\left\{\left(x_{1}, x_{2}, x_{3}\right): x_{1}=0\right\}$. Let $C$ be the set of all points in $I^{3}$ whose coordinates are all rational. If we set $X=B \cup C$, then $(X, \rho)$ satisfies the condition as follows: $2=d_{2}(B, \rho) \leqq d_{2}(X, \rho) \leqq d_{3}(X, \rho) \leqq \mu \operatorname{dim}(X, \rho) \leqq \operatorname{dim} X=2$.

REMARK 2. It is to be noticed that even if $(X, \rho)$ is any metric space, (i) $\operatorname{dim} X=1$ implies $d_{2}(X, \rho)=1$ and (ii) $d_{2}(X, \rho)=0$ implies $\operatorname{dim} X=0$ (see Nagami-Roberts [15]).

\section{Spaces $\left(X_{n}, \rho\right)$ with $d_{2}=[n / 2]$ and $\operatorname{dim} \geqq n-1$.}

LEMMA 8. Let $F_{1}, F_{2}, \ldots$ be a sequence of closed sets of a metric space $X$ with $\operatorname{dim} F_{i}=n_{i}$. Let $C_{1}, C_{1}^{\prime} ; \ldots ; C_{m}, C_{m}^{\prime}$ be $m$ disjoint pairs of closed sets of $X$. Then there exist closed sets $B_{1}, \ldots, B_{m}$ such that

(i) for each $i B_{i}$ separates $C_{i}$ and $C_{i}^{\prime}$,

(ii) for each $j$ and for each sequence $1 \leqq i_{1}<i_{2}<\cdots<i_{t} \leqq \min \{n+1, m\}$,

$$
\operatorname{dim}\left(B_{i_{1}} \cap \cdots \cap B_{i_{t}} \cap F_{j}\right) \leqq n_{j}-t .
$$

See Morita [12, Theorem 9.1].

Construction of $\left(X_{n}, \rho\right)$. Let $\left(K_{n}, \rho\right)$ be a Cantor $n$-manifold with $n \geqq 3$. Put $m=[n / 2]+1$. By compactness of $K_{n}$ there exists a sequence of $m$ disjoint pairs of closed sets of $K_{n}$, say $C_{11}, C_{11}^{\prime} ; \ldots ; C_{1 m}, C_{1 m}^{\prime}: C_{21}, C_{21}^{\prime} ; \ldots ; C_{2 m}, C_{2 m}^{\prime}: \ldots$, such that for any $m$ disjoint pairs of closed sets $C_{1}, C_{1}^{\prime} ; \ldots ; C_{m}, C_{m}^{\prime}$ there exists $i$ with $C_{j} \subset C_{i j}$ and $C_{j}^{\prime} \subset C_{i j}^{\prime}$ for $j=1, \ldots, m$. By Lemma 8 there exist closed sets $B_{11}, \ldots$, $B_{1 m}$ such that

(i) for each $i B_{1 i}$ separates $C_{1 i}$ and $C_{1 i}^{\prime}$,

(ii) $\operatorname{dim} B_{1} \leqq n-m$ where $B_{1}=\bigcap_{j=1}^{m} B_{1 j}$.

By repeated application of Lemma 8 there exist closed sets $B_{21}, \ldots, B_{2 m}$ such that

(i) for each $i B_{2 i}$ separates $C_{2 i}$ and $C_{2 i}^{\prime}$,

(ii) $\operatorname{dim} B_{2} \leqq n-m$ where $B_{2}=\bigcap_{j=1}^{m} B_{2 j}$,

(iii) $B_{1} \cap B_{2}=\varnothing$.

Continuing such process we get finally a sequence of closed sets $B_{i j}, i=1,2, \ldots$, $j=1, \ldots, m$, which have the following property:

(i) $B_{i j}$ separates $C_{i j}$ and $C_{i j}^{\prime}$ for each $i$ and $j$.

(ii) $\operatorname{dim} B_{i} \leqq n-m$ for $i=1,2, \ldots$, where $B_{i}=\bigcap_{j=1}^{m} B_{i j}$.

(iii) $B_{i} \cap B_{j}=\varnothing$ if $i \neq j$. 
If we set

$$
X_{n}=K_{n}-\bigcup_{i=1}^{\infty} B_{i}
$$

then we have the space $\left(X_{n}, \rho\right)$.

AsSERTION 1. $d_{2}\left(X_{n}, \rho\right) \leqq[n / 2]$.

Proof. Let $C_{1}, C_{1}^{\prime} ; \ldots ; C_{m}, C_{m}^{\prime}$ be $m$ pairs of closed sets of $X_{n}$ such that $\rho\left(C_{i}, C_{i}^{\prime}\right)>0$ for $i=1, \ldots, m$. Since their closures $\bar{C}_{1}, \bar{C}_{1}^{\prime} ; \ldots ; \bar{C}_{m}, \bar{C}_{m}^{\prime}$ in $K_{n}$ constitute $m$ disjoint pairs of closed sets of $K_{n}$, there exists $i$ such that $\bar{C}_{j} \subset C_{i j}$ and $\bar{C}_{j}^{\prime} \subset C_{i j}^{\prime}$ for $j=1, \ldots, m$. Then $B_{i 1} \cap X_{n}, \ldots, B_{i m} \cap X_{n}$ are closed sets of $X_{n}$ such that $B_{i j} \cap X_{n}$ separates $C_{j}$ and $C_{j}^{\prime}$ for $j=1, \ldots, m$.

$$
\bigcap_{j=1}^{m}\left(B_{i j} \cap X_{n}\right)=B_{i} \cap X_{n}=\varnothing,
$$

and hence we have $d_{2}\left(X_{n}, \rho\right) \leqq m-1=[n / 2]$.

ASSERTION 2. $d_{2}\left(X_{n}, \rho\right) \geqq[n / 2]$.

Proof. If $G$ is a nonempty open set of $K_{n}$, then $\operatorname{dim} G=n$. Since

$$
\operatorname{dim}\left(\bigcup B_{i}\right) \leqq n-m<n, \quad G-\left(\bigcup B_{i}\right) \neq \varnothing
$$

and hence $G \cap X_{n} \neq \varnothing$. Thus $X_{n}$ is dense in $K_{n}$. Assume $d_{2}\left(X_{n}, \rho\right)=t<[n / 2]$. Take a defining system $\bar{D}_{1}, \bar{D}_{1}^{\prime} ; \ldots ; \bar{D}_{t+1}, \bar{D}_{t+1}^{\prime}$ of $K_{n}$ such that

(i) each $D_{i}$ and $D_{i}^{\prime}$ are open in $K_{n}$,

(ii) for any closed sets $A_{i}, i=1, \ldots, t+1$, separating $\bar{D}_{i}$ and $\bar{D}_{i}^{\prime}$,

$$
\operatorname{dim}\left(\bigcap_{i=1}^{t+1} A_{i}\right) \geqq n-(t+1) .
$$

Set $C_{i}=\bar{D}_{i} \cap X_{n}$ and $C_{i}^{\prime}=\bar{D}_{i}^{\prime} \cap X_{n}$. Then it is easy to see that $\bar{C}_{i}=\bar{D}_{i}$ and $\bar{C}_{i}^{\prime}=\bar{D}_{i}^{\prime}$, since $X_{n}$ is dense in $K_{n}$. Set

$$
\varepsilon=\min \left\{\rho\left(C_{i}, C_{i}^{\prime}\right): i=1, \ldots, t+1\right\} .
$$

Take open sets $U_{i}, i=1, \ldots, t+1$, of $K_{n}$ such that

$$
\begin{aligned}
\left\{x: x \in X_{n}, \rho\left(x, C_{i}\right)<\varepsilon / 4\right\} & \subset U_{i} \cap X_{n} \subset \bar{U}_{i} \cap X_{n} \\
& \subset X_{n}-\left\{x: x \in X_{n}, \rho\left(x, C_{i}^{\prime}\right)<\varepsilon / 4\right\} \text { for each } i, \\
& \bigcap_{i=1}^{t+1}\left(\left(\bar{U}_{i}-U_{i}\right) \cap X_{n}\right)=\varnothing .
\end{aligned}
$$

It is easy to see that $\bar{U}_{i}-U_{i}$ thus chosen separates $\bar{C}_{i}=\bar{D}_{i}$ and $\bar{C}_{i}^{\prime}=\bar{D}_{i}^{\prime}$ for each $i$. Hence

$$
\operatorname{dim} B \geqq n-(t+1) \geqq n-[n / 2] \quad \text { where } \quad B=\bigcap_{i=1}^{t+1}\left(\bar{U}_{i}-U_{i}\right)
$$


On the other hand

$$
\operatorname{dim} B \leqq \operatorname{dim}\left(\bigcup B_{i}\right) \leqq n-m=n-[n / 2]-1
$$

because $B \cap X_{n}=\varnothing$, which is a contradiction.

ASSERTION 3. $\operatorname{dim} X_{n} \geqq n-1$.

Proof. Since $\operatorname{dim} B_{i} \leqq n-m=n-[n / 2]-1 \leqq n-1$, we have $\operatorname{dim} X_{n} \geqq n-1$ at once by Theorem 1 .

5. Spaces $\left(Y_{n}, \rho\right)$ with $\mu \operatorname{dim}=[n / 2]$ and $\operatorname{dim} \geqq n-1$.

LEMma 9. $(X, \rho)$ has $\mu \operatorname{dim}(X, \rho) \leqq n$ if and only if there exists a sequence of locally finite closed coverings $\mathscr{F}_{i}, i=1,2, \ldots$, such that

(i) $\operatorname{mesh} \mathscr{F}_{i}<1 / i$ for any $i$,

(ii) ord $\mathscr{F}_{i} \leqq n+1$ for any $i$.

This is verified at once by Lemma 7 .

LEMMA 10. Let $X$ be a metric space with $\operatorname{dim} X \leqq n$ and $B_{1}, B_{2}, \ldots$ a sequence of closed sets of $X$ with $\operatorname{dim} B_{i}=n_{i}$, where $B_{1}=X$. Let $\varepsilon$ be an arbitrary positive number. . Then there exists a locally finite closed covering $\mathscr{F}=\left\{F_{\alpha}: \alpha \in \Lambda\right\}$ which satisfies the following conditions:

(i) $\operatorname{mesh} \mathscr{F}<\varepsilon$.

(ii) For any $i$ ord $\mathscr{F} \mid B_{i} \leqq n_{i}+1$.

(iii) For any $i$, any $j \leqq n_{i}+2$ and any $j$ different indices $\alpha(1), \ldots, \alpha(j)$ of $\Lambda$,

$$
\operatorname{dim} \bigcap_{k=1}^{j}\left(F_{\alpha(k)} \cap B_{i}\right) \leqq n_{i}-j+1 .
$$

This is proved essentially in Nagami [13, Theorem 3.6].

Construction of $\left(Y_{n}, \rho\right)$. Let $\left(K_{n}, \rho\right)$ be a Cantor $n$-manifold, $n \geqq 3$. Set $m=[n / 2]$ +2. By Lemma 10 there exists a locally finite closed covering $\mathscr{F}_{1}=\left\{F_{\alpha}: \alpha \in \Lambda_{1}\right\}$ of $K_{n}$ such that (i) mesh $\mathscr{F}_{i}<1$, (ii) ord $\mathscr{F}_{1} \leqq n+1$, and (iii) $\operatorname{dim} B_{1} \leqq n-m+1$ where $B_{1}=\left\{x:\right.$ ord $\left.\left(x, \mathscr{F}_{1}\right) \geqq m\right\}$ which is closed by the local finiteness of $\mathscr{F}_{1}$. Then ord $\mathscr{F}_{1} \mid K_{n}-B_{1}<m$.

By Lemma 10 again there exists a locally finite closed covering

$$
\mathscr{F}_{2}=\left\{F_{\alpha}: \alpha \in \Lambda_{2}\right\}
$$

of $K_{n}$ such that (i) mesh $\mathscr{F}_{2}<1 / 2$, (ii) ord $\mathscr{F}_{2} \leqq n+1$, (iii) $\operatorname{dim} B_{2} \leqq n-m+1$ where $B_{2}=\left\{x:\right.$ ord $\left.\left(x, \mathscr{F}_{2}\right) \geqq m\right\}$, and (iv) $\operatorname{dim} \bigcap_{k=1}^{j}\left(F_{\alpha(k)} \cap B_{1}\right) \leqq \operatorname{dim} B_{1}-j+1$ for any $j \leqq \operatorname{dim} B_{1}+2$ and any $j$ different indices $\alpha(1), \ldots, \alpha(j)$ of $\Lambda_{2}$. To show that the last condition (iv) implies $B_{1} \cap B_{2}=\varnothing$, set $\operatorname{dim} B_{1}=n_{1}$. Take $n_{1}+2$ different indices $\alpha(1), \ldots, \alpha\left(n_{1}+2\right)$ of $\Lambda_{2}$. Then

$$
\operatorname{dim} \bigcap_{k=1}^{n_{1}+2}\left(F_{\alpha(k)} \cap B_{1}\right) \leqq n_{1}-\left(n_{1}+2\right)+1=-1 .
$$

Hence we have

$$
B_{1} \cap\left\{x: \text { ord }\left(x, \mathscr{F}_{2}\right) \geqq n_{1}+2\right\}=\varnothing
$$


Since

we have $B_{1} \cap B_{2}=\varnothing$.

$$
\begin{aligned}
n_{1}+2 & \leqq(n-m+1)+2 \leqq n-([n / 2]+2)+3 \\
& =n-[n / 2]+1 \leqq(2[n / 2]+1)-[n / 2]+1 \\
& =[n / 2]+2=m,
\end{aligned}
$$

Repeating such procedure we have a sequence of locally finite closed coverings $\mathscr{F}_{i}, i=1,2, \ldots$, which satisfy the following conditions:

(i) For each $i$, mesh $\mathscr{F}_{i}<1 / i$.

(ii) For each $i, \operatorname{dim} B_{i} \leqq n-m+1$ where $B_{i}$ is a closed set defined by

$$
B_{i}=\left\{x: \text { ord }\left(x, \mathscr{F}_{i}\right) \geqq m\right\} .
$$

(iii) $B_{i}, i=1,2, \ldots$, are mutually disjoint.

We set $Y_{n}=K_{n}-\bigcup B_{i}$. Then $\left(Y_{n}, \rho\right)$ is the desired space.

ASSERTION 1. $\operatorname{dim} Y_{n} \geqq n-1$.

Proof. Since

$$
\operatorname{dim} B_{i} \leqq n-m+1=n-[n / 2]-1 \leqq n-1,
$$

the assertion is true by Theorem 1 .

AsSERTION 2. $\mu \operatorname{dim}\left(Y_{n}, \rho\right) \leqq[n / 2]$.

Proof. Since ord $\mathscr{F}_{i} \mid Y_{n} \leqq$ ord $\mathscr{F}_{i} \mid K_{n}-B_{i} \leqq m-1=([n / 2]+2)-1=[n / 2]+1$, the assertion is true by Lemma 9 .

AsSERTION 3. $\operatorname{dim} Y_{n} \leqq n-1$ when $n$ is odd.

Proof. Since $\operatorname{dim} Y_{n} \leqq 2 \mu \operatorname{dim}\left(Y_{n}, \rho\right)$ by Theorem 3, we have

$$
\operatorname{dim} Y_{n} \leqq 2[n / 2]=2((n-1) / 2)=n-1 .
$$

ASSERTION 4. $\mu \operatorname{dim}\left(Y_{n}, \rho\right) \geqq[n / 2]$.

Proof. Assume the contrary. Then

$$
\operatorname{dim} Y_{n} \leqq 2 \mu \operatorname{dim}\left(Y_{n}, \rho\right) \leqq 2([n / 2]-1) \leqq n-2,
$$

a contradiction.

Thus $\left(Y_{n}, \rho\right)$ satisfies (i) $\operatorname{dim} Y_{n} \geqq n-1$ and (ii) $\mu \operatorname{dim}\left(Y_{n}, \rho\right)=[n / 2]$. Furthermore when $n$ is odd, $\operatorname{dim} Y_{n}=n-1$.

REMARK 3. It is to be noted that for $X_{n}$ and $Y_{n}$ obtained by replacing $K_{n}$ with $I^{n}, \operatorname{dim} X_{n}=\operatorname{dim} Y_{n}=n-1$ for any $n$, because of the fact that $I^{n}-X_{n}$ and $I^{n}-Y_{n}$ are dense in $I^{n}$, and the invariance theorem of domain.

REMARK 4. Note that the existence of a sequence of open coverings $\mathscr{U}_{i}$, $i=1,2, \ldots$, with ord $\mathscr{U}_{i} \leqq n+1$ and lim mesh $\mathscr{U}_{i}=0$ does not characterize dimension. Thus it is natural to seek an additional condition upon $\mathscr{U}_{i}$ with which the existence of the sequence does characterize dimension. Dowker-Hurewicz [2], Nagata [17] and Nagami [14] considered such a condition. This type of characterization theorem is one of the main foundations on which modern dimension theory has been built up. Vopěnka [22] gave a simple condition: " $\mathscr{U}_{i+1}<$ (refines) $\mathscr{U}_{\mathfrak{\imath}}$ for each $i$ ". Recently Nagami-Roberts [16, Theorem 3] refined Vopěnka's 
theorem, weakening the mesh condition. But our proof contains an error. The definition of $V_{\alpha}$ in [16, line 15, p. 157] is not adequate. Let us take this opportunity to give a correct proof as follows:

TheOREM 6. A metric space $X$ has $\operatorname{dim} X \leqq n$ if there exists a sequence $\mathscr{U}_{1}>\mathscr{U}_{2}$ $>\cdots$ of open coverings $\mathscr{U}_{i}$ of $X$ such that

(i) for each $x \in X,\left\{\mathrm{St}\left(x, \mathscr{U}_{i}^{\Delta}\right): i=1,2, \ldots\right\}$ is a local base of $x$,

(ii) ord $\mathscr{U}_{i} \leqq n+1$.

Proof. Set

$$
\mathscr{U}_{i}=\left\{U\left(\alpha_{i}\right): \alpha_{i} \in A_{i}\right\}, \quad i=1,2, \ldots
$$

Let $f_{i}^{i+1}: A_{i+1} \rightarrow A_{i}$ be a function such that $f_{i}^{i+1}\left(\alpha_{i+1}\right)=\alpha_{i}$ yields $U\left(\alpha_{i+1}\right) \subset U\left(\alpha_{i}\right)$. For each pair $i<j$ let $f_{i}^{j}=f_{i}^{i+1} \cdots f_{j-1}^{j}$ and $f_{i}^{i}$ be the identity mapping. Let $\mathscr{G}$ be an arbitrary finite open covering of $X$. Set

$$
X_{i}=\bigcup\left\{U\left(\alpha_{i}\right): \operatorname{St}\left(U\left(\alpha_{i}\right), \mathscr{U}_{i}\right) \text { refines } \mathscr{G}\right\} .
$$

Then by the condition (i) $\left\{X_{1}, X_{2}, \ldots\right\}$ is an open covering of $X$. Set $X_{0}=\varnothing$. Set

$$
\begin{aligned}
& B_{i}=\left\{\alpha_{i}: U\left(\alpha_{i}\right) \cap X_{i} \neq \varnothing\right\}, \\
& C_{i}=\left\{\alpha_{i}: \alpha_{i} \in B_{i}, U\left(\alpha_{i}\right) \cap\left(\bigcup_{j<i} X_{j}\right)=\varnothing\right\}, \\
& D_{i}=\left\{\alpha_{i}: \alpha_{i} \in B_{i}, U\left(\alpha_{i}\right) \cap\left(\bigcup_{j<i} X_{j}\right) \neq \varnothing\right\} .
\end{aligned}
$$

Then $B_{i} \subset A_{i}, B_{1}=C_{1}, B_{i}=C_{i} \cup D_{i}$ and $C_{i} \cap D_{i}=\varnothing$.

For every $i<j$ and every $\alpha_{i} \in C_{i}$ set

$$
D_{j}\left(\alpha_{i}\right)=\left(\bigcap_{k=i+1}^{j}\left(f_{k}^{j}\right)^{-1}\left(D_{k}\right)\right) \cap\left(f_{i}^{j}\right)^{-1}\left(\alpha_{i}\right) .
$$

Then

(i) $f_{k}^{j}\left(D_{j}\left(\alpha_{i}\right)\right) \subset D_{k}\left(\alpha_{i}\right), i<k \leqq j$,

(ii) $D_{j}=\bigcup\left\{D_{j}\left(\alpha_{i}\right): \alpha_{i} \in C_{i}, i<j\right\}$.

For every $\alpha_{i} \in C_{i}$ let

$$
V\left(\alpha_{i}\right)=\left(U\left(\alpha_{i}\right) \cap X_{i}\right) \cup\left(\bigcup\left\{U\left(\alpha_{j}\right) \cap X_{j}: \alpha_{j} \in D_{j}\left(\alpha_{i}\right), i<j\right\}\right) .
$$

Let us show that

$$
\mathscr{V}=\left\{V\left(\alpha_{i}\right): \alpha_{i} \in C_{i}, i=1,2, \ldots\right\}
$$

is an open covering of $X$ such that $\mathscr{V}$ refines $\mathscr{G}$ and ord $\mathscr{V} \leqq n+1$, which will prove $\operatorname{dim} X \leqq n$.

Let $x$ be an arbitrary point of $X$. Since $X_{0}=\varnothing$, there exists $i$ with $x \in X_{i}-\bigcup_{j<i} X_{j}$. Take $\alpha_{i} \in B_{i}$ with $x \in U\left(\alpha_{i}\right)$. When $\alpha_{i} \in C_{i}, x \in U\left(\alpha_{i}\right) \cap X_{i} \subset V\left(\alpha_{i}\right)$. When $\alpha_{i} \in D_{i}$, there exist $j<i$ and $\alpha_{j} \in C_{j}$ such that $\alpha_{i} \in D_{i}\left(\alpha_{j}\right)$. Then $x \in U\left(\alpha_{i}\right) \cap X_{i} \subset V\left(\alpha_{j}\right)$. Thus $\mathscr{V}$ is an open covering of $X$. 
Let $i$ be an arbitrary positive integer and $\alpha_{i}$ an arbitrary index in $C_{i}$. Since $\varnothing \neq U\left(\alpha_{i}\right) \cap X_{i} \subset V\left(\alpha_{i}\right) \subset U\left(\alpha_{i}\right)$, there exists $\beta_{i} \in A_{i}$ such that $U\left(\beta_{i}\right) \cap U\left(\alpha_{i}\right) \cap X_{i} \neq \varnothing$ and $\operatorname{St}\left(U\left(\beta_{i}\right), \mathscr{U}_{i}\right)$ refines $\mathscr{G}$. Thus $V\left(\alpha_{i}\right)$ refines $\mathscr{G}$ and hence $\mathscr{V}$ refines $\mathscr{G}$.

To prove ord $\mathscr{V} \leqq n+1$ assume the contrary. Then there exist a point $x$ and $n+2$ indices $\alpha^{1}, \ldots, \alpha^{n+2}$ such that

(i) $\alpha^{i} \in C_{m_{i}}, i=1, \ldots, n+2$,

(ii) $x \in V\left(\alpha^{i}\right), i=1, \ldots, n+2$.

Let $k$ be the smallest integer such that $x \in X_{k}-\bigcup_{j<k} X_{j}$. Every $m_{i}$ is less than or equal to $k$. For every $\alpha^{i}$ there exist $j(i)$ with $j(i) \geqq k$ and $\beta^{i} \in D_{j(i)}\left(\alpha^{i}\right)$ such that $x \in U\left(\beta^{i}\right)$. Set $\gamma^{i}=f_{k}^{j(i)}\left(\beta^{i}\right)$. Then (i) $x \in U\left(\gamma^{i}\right)$, (ii) $\gamma^{i} \in D_{k}\left(\alpha^{i}\right)$ if $m_{i}<k$ and (iii) $\gamma^{i}=\alpha^{i}$ if $m_{i}=k$. Since $\gamma^{i}, i=1, \ldots, n+2$, are all different from one another by our construction, ord $\left(x, \mathscr{U}_{k}\right) \geqq n+2$, a contradiction. Hence ord $\mathscr{V} \leqq n+1$ and the proof is finished.

6. Spaces $\left(Z_{n}, \sigma_{i}\right)$ illustrating the dependence of $\mu$ dim and $d_{2}$ on the metric.

LEMMA 11. If $(X, \rho)$ is a metric space with $\operatorname{dim} X=n$, then there exists an equivalent metric $\rho^{\prime}$ to $\rho$ such that $d_{2}\left(X, \rho^{\prime}\right)=n$.

Proof. Since $\operatorname{dim} X=n$, there exists a defining system of $n$ pairs $C_{1}, C_{1}^{\prime} ; \cdots$; $C_{n}, C_{n}^{\prime}$. Let $f_{1}, \ldots, f_{n}$ be real-valued mappings of $X$ such that

(i) $0 \leqq f_{i}(x) \leqq 1$ for any $i$ and any $x \in X$,

(ii) $f_{i}(x)=0$ for any $i$ and any $x \in C_{i}$,

(iii) $f_{i}(x)=1$ for any $i$ and any $x \in C_{i}^{\prime}$.

Set

$$
\rho^{\prime}(x, y)=\rho(x, y)+\sum_{i=1}^{n}\left|f_{i}(x)-f_{i}(y)\right| .
$$

Then $\rho^{\prime}$ is an equivalent metric to $\rho$ and $\rho^{\prime}\left(C_{i}, C_{i}^{\prime}\right)>0$ for each $i$. Thus we have $d_{2}\left(X, \rho^{\prime}\right) \geqq n$ and hence $d_{2}\left(X, \rho^{\prime}\right)=n$.

Construction of $Z_{n}, n \geqq 2$. Set $m=[(n+1) / 2]+1$. In every $\left(I^{i}, \rho_{i}\right), i=m, m+1$, $\ldots, n+1$, we construct $\left(Y_{i}, \rho_{i}\right)$ as in the preceding section. Then $\mu \operatorname{dim}\left(Y_{i}, \rho_{i}\right)$ $\leqq[i / 2] \leqq[(n+1) / 2]$ and $\operatorname{dim} Y_{i}=i-1$ for $i=m, \ldots, n+1$. We assume here that $\rho_{i}\left(I^{i}\right) \leqq 1$ for $i=m, \ldots, n+1$. Take a metric $\rho_{i}^{\prime}$ equivalent to $\rho_{i}$ as in Lemma 11 such that $d_{2}\left(Y_{i}, \rho_{i}^{\prime}\right)=i-1$. Then $\mu \operatorname{dim}\left(Y_{i}, \rho_{i}^{\prime}\right)=d_{3}\left(Y_{i}, \rho_{i}^{\prime}\right)=i-1$ are automatically true for $i=m, \ldots, n+1$. By the construction of $\rho_{i}^{\prime}$ in Lemma $11 \rho_{i}^{\prime}$ satisfies $\rho_{i}^{\prime}\left(Y_{i}\right) \leqq i+1$.

$Z_{n}$ is merely the disjoint sum of $Y_{m}, Y_{m+1}, \ldots, Y_{n+1}$. The topology of $Z_{n}$ is defined in such a way that a subset $G$ of $Z_{n}$ is open if and only if $G \cap Y_{i}$ is open in $Y_{i}$ for $i=m, \ldots, n+1$. Then $Z_{n}$ is a metric space. Define for $i=m, \ldots, n+1$ the metrics $\sigma_{i}$ of $Z_{n}$ as follows:

(i) $\sigma_{i} \mid Y_{j}=\rho_{j}$ if $i \neq j$.

(ii) $\sigma_{i} \mid Y_{i}=\rho_{i}^{\prime}$.

(iii) $\sigma_{i}(x, y)=n+2$ if for any $j=m, \ldots, n+1, x$ and $y$ are not in the same $Y_{j}$. $\sigma_{m}, \ldots, \sigma_{n+1}$ are equivalent metrics which give the preassigned topology of $Z_{n}$. 
ASSERTION 1. $\operatorname{dim} Z_{n}=n$.

Proof. $\operatorname{dim} Z_{n}=\max \left\{\operatorname{dim} Y_{i}: i=m, \ldots, n+1\right\}=n$.

ASSERTION 2. $\mu \operatorname{dim}\left(Z_{n}, \sigma_{i}\right)=d_{2}\left(Z_{n}, \sigma_{i}\right)=d_{3}\left(Z_{n}, \sigma_{i}\right)=i-1$ for $i=[(n+1) / 2]+1$, $\ldots, n+1$.

Proof. If $j \neq i$, then $\mu \operatorname{dim}\left(Y_{j}, \sigma_{i}\right)=\mu \operatorname{dim}\left(Y_{j}, \rho_{j}\right) \leqq[(n+1) / 2]$. Since

$\mu \operatorname{dim}\left(Y_{i}, \sigma_{i}\right)=d_{2}\left(Y_{i}, \sigma_{i}\right)=d_{3}\left(Y_{i}, \sigma_{i}\right)=\mu \operatorname{dim}\left(Y_{i}, \rho_{i}^{\prime}\right)=i-1 \geqq[(n+1) / 2]$,

we have

$i-1=d_{2}\left(Z_{n}, \sigma_{i}\right) \leqq d_{3}\left(Z_{n}, \sigma_{i}\right) \leqq \mu \operatorname{dim}\left(Z_{n}, \sigma_{i}\right)$

$=\max \left\{\mu \operatorname{dim}\left(Y_{m}, \rho_{m}\right), \ldots, \mu \operatorname{dim}\left(Y_{i-1}, \rho_{i-1}\right), \mu \operatorname{dim}\left(Y_{i}, \rho_{i}^{\prime}\right)\right.$, $\left.\mu \operatorname{dim}\left(Y_{i+1}, \rho_{i+1}\right), \ldots, \mu \operatorname{dim}\left(Y_{n+1}, \rho_{n+1}\right)\right\}=i-1$.

Thus the assertion is proved.

7. A space $(R, \rho)$ with $d_{2}=2, \mu \operatorname{dim}=3, \operatorname{dim}=4$.

First let us construct a space $(S, \sigma)$ with $d_{2}(S, \sigma)=2$ and $\mu \operatorname{dim}(S, \sigma)=\operatorname{dim} S=3$.

Construction of $(S, \sigma)$. $(S, \sigma)$ will be a subset of

$$
\left(I^{4}=\left\{\left(x_{1}, \ldots, x_{4}\right): 0 \leqq x_{i} \leqq 1, i=1, \ldots, 4\right\}, \sigma\right),
$$

where $\sigma$ is Euclidean metric on $I^{4}$. Let $C_{i j}, C_{i j}^{\prime}, i=1,2, \ldots, j=1,2,3$, be disjoint pairs of closed sets of $I^{4}$ such that for any three disjoint pairs of closed sets $C_{1}, C_{1}^{\prime} ; C_{2}, C_{2}^{\prime} ; C_{3}, C_{3}^{\prime}$, there exists $i$ with $C_{j} \subset C_{i j}$ and $C_{j}^{\prime} \subset C_{i j}^{\prime}$ for $j=1,2,3$. Let $\pi$ be a prime number with $5 \leqq \pi$. Consider an open covering $\mathscr{D}(\pi)$ of the unit interval $[0,1]$ consisting of overlapping intervals $[0,2 / \pi),((\pi-2) / \pi, 1]$ and $((2 k-1) / \pi,(2 k+2) / \pi), k=1, \ldots,(\pi-3) / 2$. Define an open covering $\mathscr{E}(\pi)$ of $I^{4}$ as follows:

$$
\begin{aligned}
\mathscr{E}(\pi) & =\left\{D_{1} \times D_{2} \times D_{3} \times D_{4}: D_{1}, \ldots, D_{4} \in \mathscr{D}(\pi)\right\} \\
& =\left\{E_{\lambda}: \lambda \in \Lambda(\pi)\right\} .
\end{aligned}
$$

Let $\pi_{i j}, i=1,2, \ldots, j=1,2,3$, be prime numbers which are different from each other and satisfy the following conditions:

(i) $5 \leqq \pi_{i j}$ for every $i$ and $j$.

(ii) $\max \left\{\operatorname{mesh} \mathscr{E}\left(\pi_{i j}\right): j=1,2,3\right\}<\min \left\{\sigma\left(C_{i j}, C_{i j}^{\prime}\right): j=1,2,3\right\}$ for every $i$.

Let $U_{i j}$ be the sum of all elements of $\mathscr{E}\left(\pi_{i j}\right)$ which meet $C_{i j}$. Set $B_{i j}=\bar{U}_{i j}-U_{i j}$ and $B_{i}=\bigcap_{j=1}^{3} B_{i j}$. Then $B_{i j}$ separates $C_{i j}$ and $C_{i j}^{\prime}$. Set

$$
S=I^{4}-\bigcup B_{i} \text {. }
$$

Then $(S, \sigma)$ satisfies the required equalities.

AsSERTION 1. $B_{i} \cap B_{k}=\varnothing$ if $i \neq k$.

Proof. Set

$$
L_{i j}=\left\{a / \pi_{i j}: a=1, \ldots, \pi_{i j}-1\right\} .
$$

Then $L_{i j} \cap L_{k l} \neq \varnothing$ if and only if $i=k$ and $j=l$. If $x=\left(x_{1}, \ldots, x_{4}\right)$ is a point of $B_{i j}$, then for some $t, x_{t} \in L_{i j}$. Hence $B_{i} \cap B_{k}=\varnothing$ if $i \neq k$. 
ASSERTION 2. $B_{i}$ does not meet the 2-dimensional edge of $I^{4} . B_{i}$ meets the surface of $I^{4}$ at only a finite number of points. $B_{i}$ is the sum of a finite number of segments.

This is evident from the above observation.

ASSERTION 3. $B_{i}$ is the disjoint sum of a finite number of simple closed curves and a finite number of simple arcs.

Proof. If three different lines $l_{1}, l_{2}, l_{3}$ lying in $B_{i}$ have a common point, then they lie in some hyperplane $H: x_{j}=$ constant. Since $H$ is 3-dimensional, it is now easy to see that $H \cap B_{i}$ cannot contain $l_{1}, l_{2}, l_{3}$ at the same time because (i) $\mathscr{E}\left(\pi_{i j}\right) \mid H$, $=1,2,3$, are collections of bordered blicks and (ii) $\pi_{i j}, j=1,2,3$, are different from each other.

ASSERTION 4. $d_{2}(S, \sigma)=2$ and $\operatorname{dim} S=3$.

The first equality was proved in $\$ 4$. As for the second equality see Remark 3.

ASSERTION 5. $\mu \operatorname{dim}(S, \sigma)=3$.

Proof. To show $\mu \operatorname{dim}(S, \sigma)>2$, assume that $\mu \operatorname{dim}(S, \sigma) \leqq 2$. Then there exists a finite closed (in $S$ ) covering $\mathscr{F}=\{F\}$ of $S$ which satisfies the following conditions:

(i) $\{G(F)=$ interior of $F$ with respect to $S: F \in \mathscr{F}\}$ covers $S$.

(ii) $\operatorname{mesh} \mathscr{F}<1$.

(iii) ord $\mathscr{F} \leqq 3$.

The proof for the existence of such $\mathscr{F}$ is left to the reader. Cf. Lemma 7 and also use the total boundedness of $(S, \sigma)$. Set

$$
\begin{aligned}
\mathscr{F}_{1} & =\left\{F: F \in \mathscr{F}, \bar{F} \cap\left\{x: x_{1}=0\right\} \neq \varnothing\right\}, \\
M_{1} & =\text { boundary in } I^{4} \text { of } \bigcup\left\{\bar{F}: F \in \mathscr{F}_{1}\right\} .
\end{aligned}
$$

Let $F$ be an arbitrary element of $\mathscr{F}_{1}$. Let $G^{\prime}$ be an open set of $I^{4}$ with $G^{\prime} \cap S=G(F)$. Since $\operatorname{dim} \bigcup B_{i}=1, S$ is dense in $I^{4}$. Hence $G^{\prime}-\bar{F} \neq \varnothing$ yields $\left(G^{\prime}-\bar{F}\right) \cap S \neq \varnothing$, a contradiction. Thus $G^{\prime} \subset \bar{F}$, which implies $G(F) \cap M_{1}=\varnothing$. Take an arbitrary point $x$ from $M_{1} \cap S$. Since $x \notin G(F)$ for any $F$ in $\mathscr{F}_{1}$, there exists an element $F_{0} \in \mathscr{F}-\mathscr{F}_{1}$ such that $x \in G\left(F_{0}\right)$ by the condition (i) imposed upon $\mathscr{F}$. Hence

Set

$$
\text { ord } \mathscr{F}_{1} \mid M_{1} \cap S \leqq \text { ord } \mathscr{F}-1 \leqq 2 .
$$

$$
\begin{aligned}
\mathscr{F}_{2} & =\left\{F: F \in \mathscr{F}_{1}, \bar{F} \cap\left\{x: x_{2}=0\right\} \neq \varnothing\right\}, \\
M_{2} & =\text { boundary in } M_{1} \text { of } \bigcup\left\{\bar{F} \cap M_{1}: F \in \mathscr{F}_{2}\right\}
\end{aligned}
$$

Take an arbitrary point $x^{\prime}$ from $M_{2} \cap S$. Let $y^{1}, y^{2}, \ldots$ be a sequence of points of $M_{1}-\bigcup\left\{\bar{F} \cap M_{1}: F \in \mathscr{F}_{2}\right\}$ with $\lim y^{i}=x^{\prime}$. Since $\mathscr{F}_{1}$ is finite and $\overline{\mathscr{F}}_{1}=\left\{\bar{F}: F \in \mathscr{F}_{1}\right\}$ covers $M_{1}$, we assume here without loss of generality that the sequence $\left\{y^{i}\right\}$ is contained in one $\bar{F}_{1}$ with $F_{1} \in \mathscr{F}_{1}-\mathscr{F}_{2}$. For any $i$ let $z^{i}$ be a point of $F_{1}$ with $\sigma\left(y^{i}, z^{i}\right)<\sigma\left(y^{i}, x^{\prime}\right)$. Since $\lim z^{i}=x^{\prime}, x^{\prime} \in F_{1}$. Therefore

$$
\text { ord } \mathscr{F}_{2} \mid M_{2} \cap S \leqq \text { ord } \mathscr{F}_{1} \mid M_{1} \cap S-1 \leqq 1 \text {. }
$$

Set

$$
\begin{aligned}
\mathscr{F}_{3} & =\left\{F: F \in \mathscr{F}_{2}, \bar{F} \cap\left\{x: x_{3}=0\right\} \neq \varnothing\right\}, \\
M_{3} & =\text { boundary in } M_{2} \text { of } \bigcup\left\{\bar{F} \cap M_{2}: F \in \mathscr{F}_{3}\right\}
\end{aligned}
$$


Since ord $\overline{F_{2}} \mid M_{2} \cap S=$ ord $\mathscr{F}_{2} \mid M_{2} \cap S \leqq 1$,

$$
M_{3} \cap S=\varnothing .
$$

Set

$$
T=\left\{x: x \in M_{2}, \text { ord }\left(x, \overline{\mathscr{F}_{2}}\right) \geqq 2\right\} .
$$

Then $T$ is a closed set of $I^{4}$ such that

$$
M_{3} \subset T \subset M_{2} \cap\left(\bigcup B_{i}\right) .
$$

Let $K_{1}$ and $K_{2}$ be mutually separated relatively open sets of $M_{2}$ such that

$$
\begin{aligned}
& M_{2}-M_{3}=K_{1} \cup K_{2}, \\
& K_{1} \supset M_{2} \cap\left\{x: x_{3}=0\right\}, \\
& K_{2} \supset M_{2} \cap\left\{x: x_{3}=1\right\} .
\end{aligned}
$$

Let $P, P^{\prime}, Q$ or $Q^{\prime}$ be the union of all components of $T$ which meet $\left\{x: x_{3}=0\right\}$, $\left\{x: x_{3}=1\right\},\left\{x: x_{4}=0\right\}$ or $\left\{x: x_{4}=1\right\}$, respectively. Then these four sets are closed. Let us show for instance $P$ is closed. Let $x^{0}$ be an arbitrary point of the closure of $P$ and $C_{1}, C_{2}, \ldots$ a sequence of components of $T$ such that

(i) each $C_{i}$ intersects $\left\{x: x_{3}=0\right\}$,

(ii) each $C_{i}$ contains a point $z^{i}$ with $\lim z^{i}=x^{0}$.

Since $x^{0} \in \lim \inf C_{i}, \lim \sup C_{i}$ is connected by [6, Theorem 2-101]. Since $\lim \sup C_{i}$ intersects $\left\{x: x_{3}=0\right\}$ and $\lim \sup C_{i} \subset T, \lim \sup C_{i} \subset P$. Especially $x^{0} \in P$ and hence $P$ is closed.

By Assertions 2 and $3 P \cup P^{\prime}$ and $Q \cap Q^{\prime}$ are disjoint closed sets of $T$ such that there is no continuum in $T$ between them. Hence by Lemma 2 there exists a subset $V$ of $T$ such that

(i) $V$ is open and closed in $T$,

(ii) $Q \cap Q^{\prime} \subset V$,

(iii) $V \cap\left(P \cup P^{\prime}\right)=\varnothing$.

Since $Q \cap Q^{\prime} \cap\left\{x: x_{3}=0,1\right\}=\varnothing$, there exists a subset $W$ of $M_{2}$ such that

(i) $W$ is open in $M_{2}$,

(ii) $W \cap T=V$,

(iii) $\bar{W} \cap\left\{x: x_{3}=0,1\right\}=\varnothing$.

Then

$$
\begin{aligned}
& (\bar{W}-W) \cap T=\varnothing, \\
& Q \cap Q^{\prime} \subset W, \\
& \bar{W} \cap\left(P \cup P^{\prime} \cup\left\{x: x_{3}=0,1\right\}\right)=\varnothing .
\end{aligned}
$$

Set

$$
\begin{aligned}
& M=\left(M_{3}-W\right) \cup(\bar{W}-W), \\
& G_{1}=K_{1}-\bar{W}, \\
& G_{2}=\left(K_{2} \cup W\right)-(\bar{W}-W) .
\end{aligned}
$$


Then

$$
\begin{aligned}
& M_{2}-M=G_{1} \cup G_{2}, \\
& G_{1} \cap G_{2}=\varnothing, \\
& G_{1} \supset M_{2} \cap\left\{x: x_{3}=0\right\}, \\
& G_{2} \supset M_{2} \cap\left\{x: x_{3}=1\right\} .
\end{aligned}
$$

Since $G_{1}$ and $G_{2}$ are open in $M_{2}, M$ separates $M_{2} \cap\left\{x: x_{3}=0\right\}$ and $M_{2}$ $\cap\left\{x: x_{3}=1\right\}$ in $M_{2}$.

Let us show that no component of $M$ meets both $\left\{x: x_{4}=0\right\}$ and $\left\{x: x_{4}=1\right\}$. Take an arbitrary element $F$ from $\mathscr{F}_{2}$. Set

$$
U(F)=M_{2}-\bigcup\left\{\bar{F}^{\prime}: F^{\prime} \in \mathscr{F}_{2}, F^{\prime} \neq F\right\} .
$$

Then $\left\{U(F): F \in \mathscr{F}_{2}\right\}$ is a disjoint collection of open sets of $M_{2}$. Since

$$
M_{2}-T=\bigcup\left\{U(F): F \in \mathscr{F}_{2}\right\}
$$

and $(\bar{W}-W) \cap T=\varnothing, \bar{W}-W$ is the sum of the disjoint collection:

$$
\mathscr{H}=\left\{(\bar{W}-W) \cap U(F)=H(F): F \in \mathscr{F}_{2}\right\} .
$$

Since

$$
H(F)=(\bar{W}-W)-\bigcup\left\{U\left(F^{\prime}\right): F^{\prime} \neq F, F^{\prime} \in \mathscr{F}_{2}\right\},
$$

$H(F)$ is closed and hence $\mathscr{H}$ is a disjoint collection of closed sets. Since

$$
\operatorname{mesh} \mathscr{H} \leqq \operatorname{mesh} \mathscr{F}_{2}<1 \text {, }
$$

no $H(F)$ meets both $\left\{x: x_{4}=0\right\}$ and $\left\{x: x_{4}=1\right\}$. Now $M$ is the sum of the disjoint closed sets:

$$
M \cap B_{i}, \quad i=1,2, \ldots, H(F) \in \mathscr{H} .
$$

By our construction no $M \cap B_{i}$ meets both $\left\{x: x_{4}=0\right\}$ and $\left\{x: x_{4}=1\right\}$ since $Q \cap Q^{\prime} \cap M=\varnothing$. Therefore no component of $M$ meets both $\left\{x: x_{4}=0\right\}$ and $\left\{x: x_{4}=1\right\}$ by Lemma 3 .

Consider the closed set:

$$
X=\left\{x: x_{4}=0,1\right\} \cup M .
$$

Let $X_{1}$ be the sum of $\left\{x: x_{4}=0\right\}$ and all components of $M$ which meet $\left\{x: x_{4}=0\right\}$. Let $X_{2}$ be the sum of $\left\{x: x_{4}=1\right\}$ and all components of $M$ which meet $\left\{x: x_{4}=1\right\}$. Then $X_{1}$ and $X_{2}$ are closed by the same argument as in the proof for the closedness of $P$. With the aid of Lemma 2 we can find a closed set $N$ of $M_{2}$ which separates $\left\{x: x_{4}=0\right\}$ and $\left\{x: x_{4}=1\right\}$ such that $N \cap M=\varnothing$. Thus two pairs of opposite sides of $M_{2}$ are not defining, which shows in turn three pairs of opposite sides of $M_{1}$ are not defining as can easily be seen. At last four pairs of opposite faces of $I^{4}$ are not defining, a contradiction. Hence $2<\mu \operatorname{dim}(S, \sigma)$. Since

$$
\mu \operatorname{dim}(S, \sigma) \leqq \operatorname{dim} S=3, \quad \mu \operatorname{dim}(S, \sigma)=3 .
$$


ASSERTION 6. $d_{3}(S, \sigma)=3$.

Proof. Since $\sigma$ is totally bounded, $d_{3}(S, \sigma)=\mu \operatorname{dim}(S, \sigma)=3$ by Theorem 5 .

Construction of $(R, \rho)$. Take the space $\left(Z_{4}, \sigma_{3}\right)$ constructed in the preceding section. Then $\operatorname{dim} Z_{4}=4$ and $d_{2}\left(Z_{4}, \sigma_{3}\right)=d_{3}\left(Z_{4}, \sigma_{3}\right)=\mu \operatorname{dim}\left(Z_{4}, \sigma_{3}\right)=2 . R$ is the disjoint union of $Z_{4}$ and $S$ just constructed. The metric $\rho$ on $R$ is defined as follows:

$$
\begin{aligned}
& \rho \mid Z_{4}=\sigma_{3}, \\
& \rho \mid S=\sigma, \\
& \rho(x, y)=\max \left\{\sigma_{3}\left(Z_{4}\right), \sigma(S)\right\}(\leqq 6) \text { if }\{x, y\} \text { is contained in neither } Z_{4} \text { nor } S .
\end{aligned}
$$

Then it is evident that $d_{2}(R, \rho)=2, d_{3}(R, \rho)=\mu \operatorname{dim}(R, \rho)=3$ and $\operatorname{dim} R=4$.

\section{Problems.}

Problem 1. Is it true that $\operatorname{dim} X \leqq 2 d_{2}(X, \rho)$ for all (separable) metric spaces $(X, \rho) ?$

Problem 2. Let $(X, \rho)$ be a metric space with $d_{2}(X, \rho)<\operatorname{dim} X$ and $k$ an arbitrary integer with

$$
d_{2}(X, \rho) \leqq k \leqq \operatorname{dim} X
$$

Can $X$ allow an equivalent metric $\sigma$ with $d_{2}(X, \sigma)=k$ ?

REMARK 5. Recently Roberts and his student Slaughter solved a problem analogous to Problem 2 for the case when $d_{2}$ is replaced by $\mu$ dim. (Added in proof. This paper has been accepted for publication in Fundamenta Mathematicae.)

Problem 3. Find a necessary and sufficient condition on $X$ with which $d_{2}(X, \rho)$ (or $\mu \operatorname{dim}(X, \rho))=\operatorname{dim} X$ for any metric $\rho$ agreeing with the preassigned topology of $X$.

RemarK 6. It is reported by Alexandroff [1] that K. Sitnikov got a sufficient condition: If $X$ is a subset of the $n$-dimensional Euclidean space $\left(R^{n}, \rho\right)$ such that $\operatorname{dim} X=\operatorname{dim} \bar{X}$, then $\mu \operatorname{dim}(X, \rho)=\operatorname{dim} X$.

Problem 4. Is there a space $(X, \rho)$ with $d_{3}(X, \rho)<\mu \operatorname{dim}(X, \rho)$ ?.

\section{REFERENCES}

1. P. Alexandroff, On some results in the theory of topological spaces obtained during the last twenty five years, Uspehi Mat. Nauk 15 (1960), $25-95$.

2. C. H. Dowker and W. Hurewicz, Dimensions of metric spaces, Fund. Math. 43 (1956), 83-87.

3. S. Eilenberg and E. Otto, Quelques propriétés caracteristiques de la dimension, Fund. Math. 31 (1938), 149-153.

4. O. Hanner, Retraction and extension of mappings of metric and nonmetric spaces, Ark. Mat. 2 (1952), 315-360.

5. E. Hemmingsen, Some theorems in dimension theory for normal Hausdorff spaces, Duke Math. J. 13 (1946), 495-504.

6. J. G. Hocking and G. S. Young, Topology, Addison-Wesley, Reading, Mass., 1961.

7. W. Hurewicz and H. Wallman, Dimension theory, Princeton Univ. Press, Princeton, N. J., 1941. 
8. M. Katětov, On the relations between the metric and topological dimensions, Czechoslovak Math. J. 8 (1958), 163-166.

9. R. L. Moore, Foundations of point set theory, Amer. Math. Soc. Colloq. Publ., Vol. 13, Amer. Math. Soc., Providence, R. I., 1932.

10. K. Morita, On the dimension of normal spaces. I, Japan. J. Math. 20 (1950), 5-36.

11. __ On the dimension of normal spaces. II, J. Math. Soc. Japan 2 (1950), 16-33.

12. - Normal families and dimension theory for metric spaces, Math. Ann. 128 (1954), $350-362$.

13. K. Nagami, Mappings of finite order and dimension theory, Japan. J. Math. 30 (1960), $25-54$.

14. - Note on metrizability and n-dimensionality, Proc. Japan Acad. 36 (1960), 565-570.

15. K. Nagami and J. H. Roberts, Metric-dependent dimension functions, Proc. Amer. Math. Soc. 16 (1965), 601-604.

16. —_ A note on countable-dimensional metric spaces, Proc. Japan Acad. 41 (1965), $155-158$.

17. J. Nagata, Note on dimension theory for metric spaces, Fund. Math. 45 (1958), 143-181.

18. - On a special metric and dimension, Fund. Math. 55 (1964), 181-194.

19. K. Sitnikov, An example of a 2-dimensional set in the 3-dimensional Euclidean space, which allows a deformation as small as desired in a 1-dimensional polyhedron, and some new character of the dimension of the sets in Euclidean spaces, Dokl. Akad. Nauk SSSR 66 (1949), 1059-1062.

20. - On the dimension of nonclosed sets of Euclidean space, Dokl. Akad. Nauk SSSR 83 (1952), 31-34.

21. A. H. Stone, Paracompactness and product spaces, Bull. Amer. Math. Soc. 54 (1948), 977-982.

22. P. Vopěnka, Remarks on the dimension of metric spaces, Czechoslovak Math. J. 9 (1959), 519-522.

\author{
EHIME UNIVERSITY, \\ MatsuYama, JAPAN \\ DUKe UNIVERSITY, \\ Durham, North Carolina
}

